\title{
Epidemiological Criteria to Support Breeding Tactics Against the Emerging, High-Consequence Wheat Blast Disease
}

\author{
M. Fernández-Campos, ${ }^{1}$ C. Góngora-Canul, ${ }^{1}$ S. Das, ${ }^{2}$ M. R. Kabir, ${ }^{3}$ B. Valent, ${ }^{4}$ and C. D. Cruz ${ }^{1, \dagger}$ \\ ${ }^{1}$ Department of Botany and Plant Pathology, Purdue University, West Lafayette, IN 47907, U.S.A. \\ ${ }^{2}$ Uttar Banga Krishi Viswavidyalaya, Pundibari, Coochbehar, West Bengal 736165, India \\ ${ }^{3}$ Bangladesh Wheat and Maize Research Institute, Nishapur, Dinajpur, Bangladesh \\ ${ }^{4}$ Department of Plant Pathology, Kansas State University, Manhattan, KS 66506, U.S.A.
}

\begin{abstract}
Plant disease epidemiology can make a significant contribution for cultivar selection by elucidating the principles of an epidemic under different levels of resistance. For emerging diseases as wheat blast (WB), epidemiological parameters can provide support for better selection of genetic resources. Field experiments were conducted at two locations in Bolivia in 2018-2019 to characterize the temporal dynamics of the disease on 10 cultivars with different levels of reaction to WB. Logistic models best $\left(\mathrm{R}^{2}=0.70-0.96\right)$ fit the disease progress curve in all cultivars followed by Gompertz $\left(R^{2}=0.64-0.94\right)$, providing additional evidence of a polycyclic disease. Total area under disease progress curve (tAUDPC), final

resistance and cultivar selection. Cultivars that showed a high spike AUDPC (sAUDPC) showed a high leaf AUDPC (IAUDPC). tAUPDC, $Y_{\max }$, and $r_{L^{*}}$ were positively correlated among them $(P<0.01)$ and all were negatively correlated with grain weight $(P<0.01)$. Based on the epidemiological parameters used, cultivars that showed resistance to WB were Urubó, San Pablo, and AN-120, which were previously reported to have effective resistance against the disease under field conditions. The information generated could help breeding programs to make technical decisions about relevant epidemiological parameters to consider prior to cultivar release.
\end{abstract} disease severity $\left(Y_{\max }\right)$, and logistic apparent infection rates $\left(r_{L^{*}}\right)$ were shown to be appropriate epidemiological parameters for describing
Keywords: wheat blast, modeling, epidemiology, resistance, breeding
Wheat blast (WB), caused by the fungus Magnaporthe oryzae pathotype triticum (MoT) (anamorph Pyricularia orizae pathotype triticum), is an important disease and an emerging threat to global wheat production. Under favorable conditions, WB can cause up to 100\% yield losses (Barea and Toledo 1996; Cabrera and Gutiérrez 2007; Malaker et al. 2016; Viedma 2005). MoT has ability to infect wheat leaves and spikes (Cruz and Valent 2017; Cruz et al. 2016a; Gomes et al. 2019; Igarashi et al. 1986). Wheat spike blast $\left(\mathrm{W}_{\mathrm{S}} \mathrm{B}\right)$ is the most visible symptom under field conditions, and the importance of wheat leaf blast $\left(\mathrm{W}_{\mathrm{L}} \mathrm{B}\right)$ for spike blast development is mostly unknown (Cruz and Valent 2017; Cruz et al. 2015, 2019; Góngora-Canul et al. 2020). Unfortunately, there is limited ecological and epidemiological information on this pathosystem. Under such a scenario, decision makers struggle to develop adequate WB management strategies and consequently, farmers rely heavily on fungicide applications. In Bolivia, for example, farmers could make up to three fungicide applications at the heading

${ }^{\dagger}$ Corresponding author: C. D. Cruz; cd-cruz@purdue.edu

Authors M. Fernandez-Campos and C. Góngora-Canul are co-first authors.

Funding: This project was supported by Agriculture and Food Research Initiative Competitive Grant no. 2013-68004-20378 from the United States Department National Institute of Food and Agriculture and The Ohio State University Department of Plant Pathology. Salaries and research support for C. Góngora-Canul, M. Fernández-Campos, and C. D. Cruz were provided by state and federal funds to Purdue University. Salaries and research support for S. Das and M. R. Kabir were provided by USDA/FAS Borlaug International Agricultural Technology Fellowship Program.

The author(s) declare no conflict of interest.

Accepted for publication 11 March 2020.

C 2020 The American Phytopathological Society stage to control the disease (Cruz et al. 2015). However, under current guidelines there is no compelling evidence to support general fungicide recommendations for $\mathrm{W}_{\mathrm{S}} \mathrm{B}$ management (Cruz et al. 2019), and numerous applications at heading may not be the most cost-effective management strategy. Specific knowledge of WB epidemiology, including dynamics of inoculum buildup under different cultivar and background levels, is necessary to manage the disease. For instance, the limited epidemiological research conducted in this pathosystem has only involved one or few susceptible cultivars (Gomes et al. 2019; Góngora-Canul et al. 2020).

International effort has been conducted toward the identification of sources of genetic resistance to $\mathrm{W}_{\mathrm{S}} \mathrm{B}$ (Arruda et al. 2005; Cruppe et al. 2020; Cruz et al. 2012, 2016b; Goulart and Paiva 1992; Igarashi 1990; Martínez et al. 2019; Prestes et al. 2007; Urashima et al. 1999, 2004). However, over the years, there have been limited sources of genetic resistance to the disease and the only effective genetic source for control is contained in the 2NS translocation from Aegilops ventricosa (Cruppe et al. 2020; Cruz et al. 2016b). In this sense, breeders have developed an excellent tactic, but without an adequate strategy, such tactic is at risk (Cruz and Valent 2017). Boom-and-bust cycles (Priestley 1978) appear to frequently occur after a period of widespread cultivation of wheat cultivars succumbing to WB (Cruz et al. 2016a; Vales et al. 2018). In addition, wheat cultivars are not immune to $\mathrm{WB}$, and there is no known source of genetic resistance that can entirely eliminate the effects of $\mathrm{W}_{\mathrm{L}} \mathrm{B}, \mathrm{W}_{\mathrm{S}} \mathrm{B}$, or both (Cruppe et al. 2020; Cruz and Valent 2017). Plant disease epidemiology provides a better understanding of the temporal and spatial dynamics of disease intensity in host populations (Madden et al. 2007). This epidemiological information is critical for the development of integrated disease management strategies.

We hypothesize that epidemiological parameters are relevant to support WB breeding tactics. To test this hypothesis, we focused on the following objective: to assess 10 spring wheat cultivars for WB resistance using epidemiological parameters. To this aim, $\mathrm{W}_{\mathrm{L}} \mathrm{B}$ and $\mathrm{W}_{\mathrm{S}} \mathrm{B}$ temporal dynamics were evaluated in multiple environments. 


\section{Materials and Methods}

Locations. Experiments were carried out at two locations in Bolivia during the 2018-2019 growing season, in the municipalities of Quirusillas and Bermejo. Experiments were established on 5 and 21 December 2018. At the two locations, the two experimental fields were planted and surrounded by infected seed. Both are experimental locations where wheat is not grown commercially. However, in Quirusillas, WB had been reported previously under natural conditions, and not in Bermejo, where MoT-infected seed induced disease development.

Genetic materials and crop management. Ten South American spring wheat cultivars with different levels of resistance to $\mathrm{W}_{\mathrm{S}} \mathrm{B}$ (Table 1) were planted at 70 to 80 seeds per linear meter at a depth of 2 to $3 \mathrm{~cm}$. Herbicides and insecticides were used for post-emergent management of weeds and pests. Both locations relied on supplemental irrigation to promote WB disease development.

Experimental design. Two experiments were established at each of two locations, for a total of four environments. The experimental design was a randomized complete block with four replications as block factors, and 10 treatments (cultivars) randomly assigned into blocks. The experimental units (i.e., plots) measured $2 \mathrm{~m} \times 2 \mathrm{~m}$. To minimize interplot interference, plots were separated by $1 \mathrm{~m}$ border rows of corn. At each location, experiment 1 was surrounded by susceptible plants of cultivar Atlax. Plants were grown from naturally
MoT-infected seed from a seed lot with 10\% MoT incidence (based on blotter test) and used as inoculum spreader. Meanwhile, experiment 2 was surrounded by moderately resistant plants of cultivar Urubó grown from untreated seed; however, each individual plot of the experiment was surrounded by susceptible plants of cultivar Atlax grown from MoT-infected seed and used as a source of inoculum. Consequently, separate analyses were performed by experiment and location.

Visual disease assessment and grain weight. Visual assessment of severity was used to study the epidemiology of $\mathrm{W}_{\mathrm{L}} \mathrm{B}$ and $\mathrm{W}_{\mathrm{S}} \mathrm{B}$. Severity was assessed as the percentage of diseased area within individual leaf or spike. Fifteen plants per plot and their plant organs, corresponding to F-6 (lower leaf), F-5, F-4, F-3, F-2, F-1, to F (flag leaf), and spikes (S), were individually evaluated at each assessment time. Visual estimations were taken 12 times in both experiments in Bermejo, and nine times for both experiments in Quirusillas. All plots were harvested by hand at the end of the season, and 100 seeds were weighed per plot (g 100 -seed $\left.{ }^{-1}\right)$.

Data analysis. The area under the disease progress curve (AUDPC) was calculated using the trapezoidal integration method (Campbell and Madden 1990). The total AUDPC (tAUDPC) was calculated considering $\mathrm{W}_{\mathrm{L}} \mathrm{B}$ and $\mathrm{W}_{\mathrm{S}} \mathrm{B}$ severity data. Leaf AUDPC (1AUDPC) and spike AUDPC (sAUDPC) were calculated separately,

Table 1. List of cultivars of different wheat spike blast resistance groups planted in two locations in Bolivia during the 2018-2019 season

\begin{tabular}{|c|c|c|c|c|}
\hline Number & Cultivar & Source & Wheat spike blast resistance ${ }^{\mathrm{z}}$ & Data source \\
\hline 1 & Atlax & ANAPO, Bolivia & $S$ & Cruz et al. 2019 \\
\hline 2 & Urubó & ANAPO, Bolivia & $\mathrm{R}$ & Cruppe et al. 2020 \\
\hline 3 & TBIO-Sossego & ANAPO, Bolivia & MR & Cruppe et al. 2020 \\
\hline 4 & San Pablo & ANAPO, Bolivia & $\mathrm{R}$ & Baldelomar et al. 2015 \\
\hline 5 & AN-120 & ANAPO, Bolivia & $\mathrm{R}$ & Cruppe et al. 2020 \\
\hline 6 & Motacú & CIAT, Bolivia & MR/MS & Cruz et al. 2016b; Vales et al. 2018 \\
\hline 7 & BR-18 & ANAPO, Bolivia & MR/MS & Cruz et al. $2016 \mathrm{~b}$ \\
\hline 8 & TBIO-Mestre & Biotrigo, Brazil & MR/MS & Cruppe et al. 2020 \\
\hline 9 & TBIO-Mirante & Biotrigo, Brazil & MS & Biotrigo Genética \\
\hline 10 & TBIO-Alvorada & Biotrigo, Brazil & $\mathrm{S}$ & Biotrigo Genética \\
\hline
\end{tabular}

${ }^{\mathrm{z}} \mathrm{S}$ : susceptible, R: resistant, MR: moderately resistant, and MS: moderately susceptible.

Table 2. Analysis of variance of repeated measurement analysis of wheat blast severity ( $\operatorname{Pr}>\mathrm{F})$ in different plant organs in Bermejo (location 1), Bolivia, during the 2018-2019 season

\begin{tabular}{|c|c|c|c|c|c|c|c|c|}
\hline Source of variation $\mathrm{z}$ & F-6 & F-5 & F-4 & F-3 & F-2 & F-1 & $\mathbf{F}$ & $\mathbf{S}$ \\
\hline DAE & $<0.0001$ & $<0.0001$ & $<0.0001$ & $<0.0001$ & 1.0000 & $<0.0001$ & $<0.0001$ & $<0.0001$ \\
\hline EXP & 1.0000 & $<0.0005$ & 0.0046 & 0.0024 & 0.9998 & 0.0399 & 0.6821 & 0.2545 \\
\hline $\mathrm{EXP} \times \mathrm{DAE}$ & 1.0000 & $<0.0001$ & $<0.0001$ & $<0.0001$ & 1.0000 & 0.0022 & 0.0446 & 0.3397 \\
\hline CUL & $<0.0001$ & $<0.0001$ & $<0.0001$ & $<0.0001$ & $<0.0001$ & $<0.0001$ & $<0.0001$ & $<0.0001$ \\
\hline $\mathrm{CUL} \times \mathrm{DAE}$ & $<0.0001$ & $<0.0001$ & $<0.0001$ & $<0.0001$ & $<0.0001$ & $<0.0001$ & $<0.0001$ & $<0.0001$ \\
\hline $\mathrm{EXP} \times \mathrm{CUL}$ & 1.0000 & $<0.0001$ & $<0.0001$ & $<0.0001$ & $<0.0001$ & $<0.0001$ & $<0.0001$ & $<0.0001$ \\
\hline $\mathrm{EXP} \times \mathrm{CUL} \times \mathrm{DAE}$ & 1.0000 & $<0.0001$ & $<0.0001$ & $<0.0001$ & $<0.0001$ & $<0.0001$ & 0.0010 & $<0.0001$ \\
\hline
\end{tabular}

${ }^{\mathrm{z}}$ DAE: days after emergency, EXP: experiment, CUL: cultivar.

Table 3. Analysis of variance of repeated measurement analysis of wheat blast severity ( $\operatorname{Pr}>\mathrm{F})$ in different plant organs in Quirusillas (location 2), Bolivia, during the 2018-2019 season

\begin{tabular}{|c|c|c|c|c|c|c|c|c|}
\hline Source of variation $^{\mathrm{z}}$ & F-6 & F-5 & F-4 & F-3 & F-2 & F-1 & $\mathbf{F}$ & $\mathbf{S}$ \\
\hline DAE & 0.5216 & 0.6975 & 0.0121 & 0.0003 & $<0.0001$ & $<0.0001$ & $<0.0001$ & $<0.0001$ \\
\hline EXP & 0.8455 & 0.0010 & 0.0010 & 0.0284 & 0.0252 & 0.0097 & 0.0051 & 0.0011 \\
\hline $\mathrm{EXP} \times \mathrm{DAE}$ & $\ldots$ & 0.1338 & 0.1056 & 0.0003 & 0.1865 & 0.0195 & 0.0195 & 0.1668 \\
\hline CUL & 0.0013 & $<0.0001$ & $<0.0001$ & $<0.0001$ & $<0.0001$ & $<0.0001$ & $<0.0001$ & $<0.0001$ \\
\hline $\mathrm{CUL} \times \mathrm{DAE}$ &.. & 0.2166 & $<0.0001$ & $<0.0001$ & $<0.0001$ & $<0.0001$ & $<0.0001$ & $<0.0001$ \\
\hline $\mathrm{EXP} \times \mathrm{VAR}$ & $\ldots$ & $<0.0001$ & $<0.0001$ & $<0.0001$ & $<0.0001$ & $<0.0001$ & $<0.0001$ & $<0.0001$ \\
\hline $\mathrm{EXP} \times \mathrm{CUL} \times \mathrm{DAE}$ & $\ldots$ & 0.2231 & $<0.0001$ & $<0.0001$ & $<0.0001$ & $<0.0001$ & $<0.0001$ & $<0.0001$ \\
\hline
\end{tabular}

${ }^{\mathrm{z}}$ DAE: days after emergency, EXP: experiment, CUL: cultivar. 
considering $\mathrm{W}_{\mathrm{L}} \mathrm{B}$ and $\mathrm{W}_{\mathrm{S}} \mathrm{B}$ severity, respectively. Repeated measurement analysis was performed using the PROC MIXED procedure in SAS (SAS Institute, Cary, NC) to evaluate the effect of disease severity (converted to arcsine root square transformation) among experiments (EXP), days after emergence (DAE), cultivars (CUL), and organ position (leaves and spike) for each location.

Graphical (untransformed $y$ versus $t$ or transformed $y^{*}$ versus $t$, where, $\left.y^{*}=\ln (y)\right)$ and statistical approaches were used to characterize the temporal dynamic of visual severity over time. The independent variable was DAE, and the dependent variable was WB expressed as $\mathrm{W}_{\mathrm{L}} \mathrm{B}$ or $\mathrm{W}_{\mathrm{S}} \mathrm{B}$ severity $(y / 100$, and when $y=0$ and $y$ $=100$, were corrected as $y=+0.0001$ and $y=-0.0001$, respectively). Severity was the average of all the plant organs of 15 plants. Four population growth models were fitted for 10 cultivars (Table 1) in their linearized form to describe WB epidemics: exponential $(\ln [y]$ $\left.=\ln \left(y_{0}\right)+r_{E} t\right)$, logistic $\left(\ln [y /(1-y)]=\ln \left[y_{0} /\left(1-y_{0}\right)\right]+r_{L} t\right.$, monomolecular $\left(\ln [1 /(1-y)]=\ln \left[1 /\left(1-y_{0}\right)\right]+r_{M} t\right)$, and Gompertz $(-\ln$ $\left.[-\ln (y)]=-\ln \left[-\ln \left(y_{0}\right)\right]+r_{G} t\right)($ Campbell and Madden 1990; Madden et al. 2007), where $y_{0}$ is the initial disease, $y$ the amount of the disease, $t$ is time, and $r$ is rate of disease progress. Ordinary least squares regression was performed with SAS (SAS Institute) for each possible model (Campbell and Madden 1990; Madden et al. 2007). Adequate model(s) to describe each epidemic was assessed by comparing the observed and transformed disease progress curve (DPCs), examining the coefficients of determination $\left(\mathrm{R}^{2}\right)$, root mean square error
(RMSE), and the plots of residual errors versus predicted values (Campbell and Madden 1990).

The two best selected models based on the above criteria were compared by using the delta Akaike's information criterion ( $\triangle \mathrm{AIC}$ ), which considers the (AIC) of all comparing models. AIC $=N *\left(\frac{S S}{N}\right)+2 K$, where $N$ is the number of the observations used in the model, $S S E$ is sum of square of the errors, and $K$ the number of parameters, and $\Delta \mathrm{AIC}=\mathrm{AIC}_{\mathrm{i}}-$ min AIC (Kaplan and Gürcan 2018; Mazerolle 2006; Raji et al. 2014; Renner-Martin et al. 2016).

The SAS Lsmeans statement was used within the PROC GLIMMIX procedure on tAUDPC, best fit model apparent infection rate $\left(r^{*}=\right.$ unit day $\left.^{-1}\right)$, final disease severity $\left(Y_{\max }=\%\right)$, and grain weight (g 100-seed ${ }^{-1}$ ) to obtain the contrast estimates and the associated standard error among all cultivars. This procedure was also used to detect differences in disease severity between cultivars, blocks in experiment 1 and 2 at both locations as influenced by the design of inoculum spreader by considering T-test pairwise comparison. Pearson correlation was performed to test the association between disease epidemiological parameters (tAUDPC, $r_{L^{*}}$, and $Y_{\text {max }}$ ), and grain weight. Linear regression between tAUDPC and grain weight was performed using PROG REG ALL procedure on SAS (SAS Institute) to estimate grain weight loss rate per location and experiment.
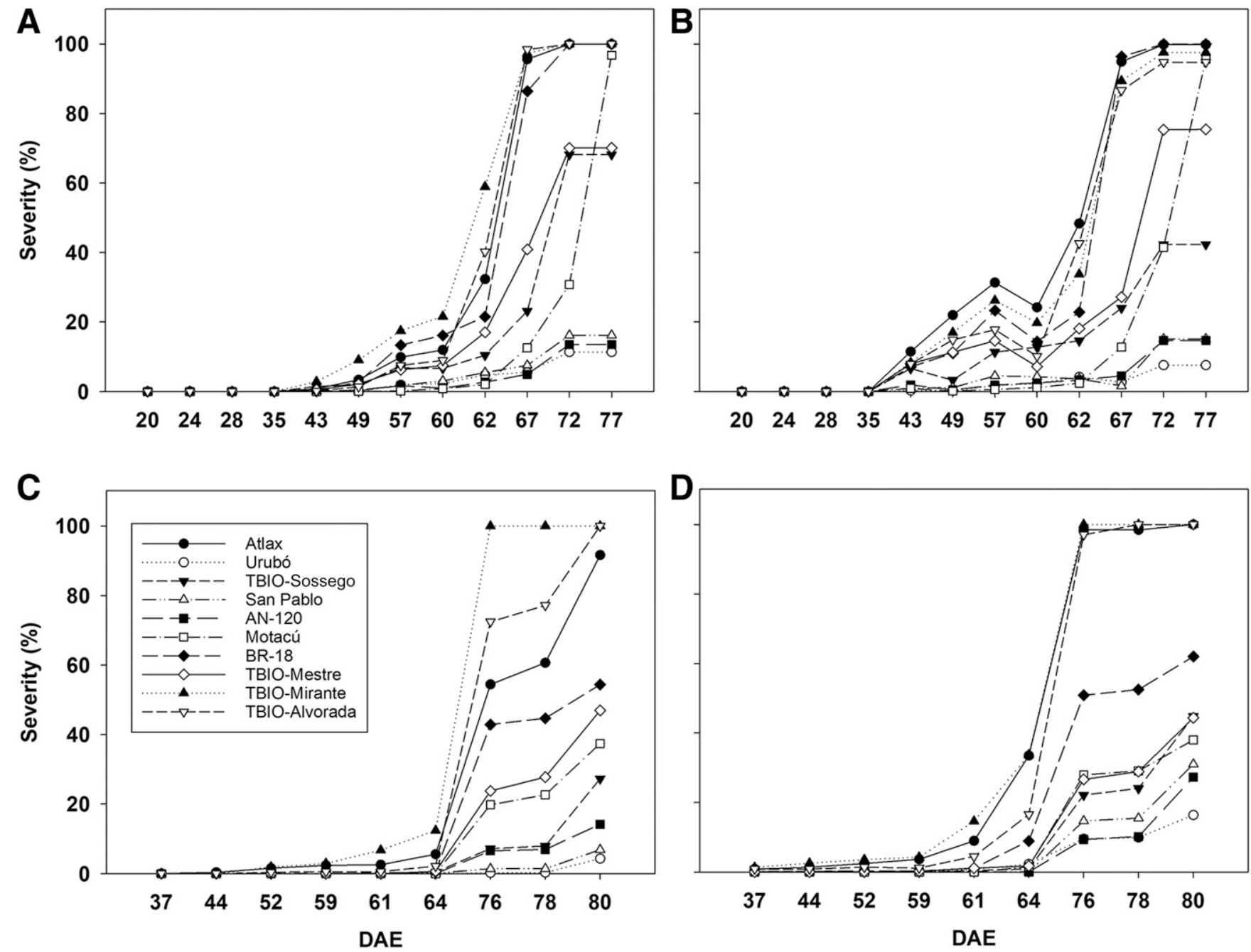

Fig. 1. Disease progress curves (DPCs) for 10 wheat cultivars (untransformed data y versus $t$ ) in epidemics induced by Magnaporthe oryzae pathotype triticum (MoT). Disease progress curves show the wheat leaf blast $\left(W_{L} B\right)$ and wheat spike blast $\left(W_{S} B\right)$ severity across all plant organs (from leaf $F-6$ to spike). Experiments 1 (A) and 2 (B) in Bermejo, (C) and (D) in Quirusillas locations conducted during the 2018-2019 growing season. 


\section{Results}

$\mathrm{W}_{\mathrm{L}} \mathrm{B}$ and $\mathrm{W}_{\mathrm{S}} \mathrm{B}$ symptoms occurred at different levels of severity in the two experiments conducted at each of the two locations, Bermejo (location 1) and Quirusillas (location 2) (Table 2 and 3) (Fig. 1). In addition, there were differences in WB resistance among cultivars, considering all plant organs (from leaf F-6 to S), with severity values ranging from 0 to $100 \%$. MoT inoculum spreader location induced differences in WB severity pressure. In general, the inoculum source

Table 4. Parameters of the pairwise comparison T-test of three susceptible cultivars to WB severity in two experiments with different spreader design at two locations in Bolivia, 2018-2019

\begin{tabular}{|c|c|c|c|c|c|c|c|c|c|}
\hline \multicolumn{10}{|c|}{ Location 1 (Bermejo) } \\
\hline Experiment & Block & Cultivar & Experiment & Block & Cultivar & Estimate & $\mathbf{S E}^{\mathbf{z}}$ & t Value & $\operatorname{Pr}>|\mathbf{t}|$ \\
\hline \multirow[t]{12}{*}{1} & \multirow[t]{3}{*}{1} & Atlax & 2 & 1 & Atlax & -0.13 & 0.019 & -6.89 & $<0.0001$ \\
\hline & & TBIO-Mirante & & & TBIO-Mirante & -0.11 & 0.020 & -5.37 & $<0.0001$ \\
\hline & & TBIO-Alvorada & & & TBIO-Alvorada & -0.13 & 0.020 & -6.37 & $<0.0001$ \\
\hline & \multirow[t]{3}{*}{2} & Atlax & & 2 & Atlax & -0.11 & 0.020 & -5.28 & $<0.0001$ \\
\hline & & TBIO-Mirante & & & TBIO-Mirante & 0.01 & 0.020 & 0.46 & 0.6436 \\
\hline & & TBIO-Alvorada & & & TBIO-Alvorada & -0.07 & 0.020 & -3.51 & 0.0004 \\
\hline & \multirow[t]{3}{*}{3} & Atlax & & 3 & Atlax & -0.01 & 0.020 & -0.46 & 0.6474 \\
\hline & & TBIO-Mirante & & & TBIO-Mirante & -0.01 & 0.020 & -0.64 & 0.5233 \\
\hline & & TBIO-Alvorada & & \multirow{4}{*}{4} & TBIO-Alvorada & -0.02 & 0.020 & -0.90 & 0.3662 \\
\hline & \multirow[t]{3}{*}{4} & Atlax & & & Atlax & -0.05 & 0.020 & -2.31 & 0.0211 \\
\hline & & TBIO-Mirante & & & TBIO-Mirante & 0.03 & 0.019 & 1.60 & 0.1102 \\
\hline & & TBIO-Alvorada & & & TBIO-Alvorada & 0.07 & 0.019 & 3.41 & 0.0006 \\
\hline \multicolumn{10}{|c|}{ Location 2 (Quirusillas) } \\
\hline \multirow[t]{12}{*}{1} & \multirow[t]{3}{*}{1} & Atlax & 2 & 1 & Atlax & -0.11 & 0.024 & -4.68 & $<0.0001$ \\
\hline & & TBIO-Mirante & & & TBIO-Mirante & -0.14 & 0.025 & -5.71 & $<0.0001$ \\
\hline & & TBIO-Alvorada & & & TBIO-Alvorada & -0.09 & 0.024 & -3.72 & 0.0002 \\
\hline & \multirow[t]{3}{*}{2} & Atlax & & 2 & Atlax & -0.28 & 0.025 & -11.38 & $<0.0001$ \\
\hline & & TBIO-Mirante & & & TBIO-Mirante & -0.11 & 0.024 & -4.49 & $<0.0001$ \\
\hline & & TBIO-Alvorada & & & TBIO-Alvorada & -0.09 & 0.025 & -3.64 & 0.0003 \\
\hline & \multirow[t]{3}{*}{3} & Atlax & & 3 & Atlax & -0.04 & 0.026 & -1.34 & 0.1789 \\
\hline & & TBIO-Mirante & & & TBIO-Mirante & -0.14 & 0.026 & -5.13 & $<0.0001$ \\
\hline & & TBIO-Alvorada & & & TBIO-Alvorada & -0.11 & 0.024 & -4.69 & $<0.0001$ \\
\hline & \multirow[t]{3}{*}{4} & Atlax & & 4 & Atlax & -0.12 & 0.025 & -4.86 & $<0.0001$ \\
\hline & & TBIO-Mirante & & & TBIO-Mirante & -0.07 & 0.026 & -2.68 & 0.0073 \\
\hline & & TBIO-Alvorada & & & TBIO-Alvorada & -0.10 & 0.026 & -4.08 & $<0.0001$ \\
\hline
\end{tabular}

${ }^{\mathrm{z}}$ SE: standard error.

Table 5. Summary of statistical parameters of four linearized temporal growth models for wheat blast severity in two locations in Bolivia 2018-2019

\begin{tabular}{|c|c|c|c|c|c|c|c|c|c|c|c|c|c|c|}
\hline \multicolumn{15}{|c|}{ Location 1 (Bermejo) } \\
\hline \multirow[b]{2}{*}{ Cultivar } & \multicolumn{7}{|c|}{ Experiment 1} & \multicolumn{7}{|c|}{ Experiment 2} \\
\hline & Model & Intercept & Slope & $\mathbf{R}^{2}$ & $\mathbf{R M S E}^{\mathbf{y}}$ & Resid. $^{z}$ & $\operatorname{Pr}>F$ & Model & Intercept & Slope & $\mathbf{R}^{2}$ & RMSE $^{y}$ & Resid. $^{\mathrm{z}}$ & $\mathbf{P}>\mathbf{F}$ \\
\hline Atlax & Logistic & -18.432 & 0.324 & 0.88 & 2.461 & $\mathrm{~S}$ & $<0.0001$ & Logistic & -16.646 & 0.292 & 0.93 & 1.666 & $\mathrm{~S}$ & $<0.0001$ \\
\hline Urubó & Logistic & -13.149 & 0.153 & 0.94 & 0.761 & S & $<0.0001$ & Gompertz & -2.892 & 0.026 & 0.94 & 0.126 & S & $<0.0001$ \\
\hline TBIO Sossego & Logistic & -14.057 & 0.196 & 0.95 & 0.923 & S & $<0.0001$ & Gompertz & -3.375 & 0.046 & 0.94 & 0.230 & S & $<0.0001$ \\
\hline San Pablo & Logistic & -13.681 & 0.162 & 0.93 & 0.923 & S & $<0.0001$ & Logistic & -12.635 & 0.149 & 0.89 & 1.057 & S & $<0.0001$ \\
\hline AN-120 & Logistic & -13.191 & 0.151 & 0.95 & 0.671 & $\mathrm{~S}$ & $<0.0001$ & Gompertz & -2.964 & 0.029 & 0.91 & 0.181 & S & $<0.0001$ \\
\hline Motacú & Logistic & -15.442 & 0.193 & 0.75 & 2.271 & NS & 0.0003 & Logistic & -14.475 & 0.19 & 0.86 & 1.539 & S & $<0.0001$ \\
\hline BR-18 & Logistic & -18.571 & 0.322 & 0.87 & 2.577 & $\mathrm{~S}$ & $<0.0001$ & Logistic & -17.964 & 0.323 & 0.88 & 2.441 & S & $<0.0001$ \\
\hline TBIO-Mestre & Logistic & -14.397 & 0.204 & 0.96 & 0.8193 & $S$ & $<0.0001$ & Logistic & -13.839 & 0.202 & 0.89 & 1.426 & $S$ & $<0.0001$ \\
\hline TBIO-Mirante & Logistic & -18.302 & 0.331 & 0.90 & 2.185 & S & $<0.0001$ & Logistic & -15.411 & 0.253 & 0.93 & 1.388 & S & $<0.0001$ \\
\hline TBIO-Alvorada & Logistic & -18.922 & 0.331 & 0.87 & 2.627 & S & $<0.0001$ & Logistic & -15.041 & 0.241 & 0.92 & 1.406 & S & $<0.0001$ \\
\hline \multicolumn{15}{|c|}{ Location 2 (Quirusillas) } \\
\hline & \multicolumn{7}{|c|}{ Experiment 1} & \multicolumn{7}{|c|}{ Experiment 2} \\
\hline Atlax & Logistic & -18.112 & 0.246 & 0.92 & 1.111 & $\mathrm{~S}$ & $<0.0001$ & Logistic & -17.969 & 0.290 & 0.81 & 2.242 & NS & 0.0009 \\
\hline Urubó & Logistic & -15.048 & 0.118 & 0.63 & 1.466 & NS & 0.0107 & Gompertz & -4.009 & 0.040 & 0.77 & 0.346 & $\mathrm{~S}$ & 0.0017 \\
\hline TBIO-Sossego & Logistic & -18.502 & 0.204 & 0.87 & 1.250 & NS & 0.0002 & Gompertz & -4.267 & 0.048 & 0.70 & 0.512 & NS & 0.0049 \\
\hline San Pablo & Logistic & -15.556 & 0.147 & 0.92 & 0.700 & $\mathrm{~S}$ & $<0.0001$ & Logistic & -20.638 & 0.234 & 0.87 & 1.454 & $\mathrm{~S}$ & 0.0002 \\
\hline AN-120 & Logistic & -18.505 & 0.189 & 0.69 & 2.031 & NS & 0.0053 & Gompertz & -4.207 & 0.041 & 0.64 & 0.494 & NS & 0.0087 \\
\hline Motacú & Logistic & -19.975 & 0.225 & 0.73 & 2.173 & NS & 0.0031 & Logistic & -21.141 & 0.248 & 0.78 & 2.087 & $\mathrm{~S}$ & 0.0014 \\
\hline BR-18 & Logistic & -21.865 & 0.262 & 0.77 & 2.309 & NS & 0.0018 & Logistic & -17.223 & 0.220 & 0.94 & 0.890 & S & $<0.0001$ \\
\hline TBIO-Mestre & Logistic & -20.804 & 0.24 & 0.70 & 2.501 & NS & 0.0045 & Logistic & -17.727 & 0.214 & 0.96 & 0.664 & $\mathrm{~S}$ & $<0.0001$ \\
\hline TBIO-Mirante & Logistic & -29.855 & 0.483 & 0.92 & 2.237 & $\mathrm{~S}$ & $<0.0001$ & Logistic & -21.062 & 0.363 & 0.81 & 2.806 & NS & 0.0009 \\
\hline TBIO-Alvorada & Logistic & -24.480 & 0.351 & 0.81 & 2.704 & NS & 0.0009 & Logistic & -20.862 & 0.338 & 0.76 & 3.080 & NS & 0.0022 \\
\hline
\end{tabular}

y RMSE: root mean square error.

${ }^{\mathrm{z}}$ Resid.: residual, S: scatter, NS: no scatter. 
on experiment 2 induced more WB severity pressure than that established on experiment 1 , for both locations $(P<0.01)$ (Table 4$)$. The reason was that on experiment 2 the susceptible Atlax plants grown from MoT-infected seed were planted closer to individual plots than the inoculum source planted around the perimeter of experiment 1 (Table 4).

WB symptoms were present in all plant organs, leaves, and spikes, at different evaluation times after crop emergence (DAE). However, in location 1, there were differences in the amount of disease over time (DAE) for each plant organ considered in the analysis (Table 2). Also, differences were found between experiments except for leaves F-6, F2 , F, and $\mathrm{S}$ (Table 2). There were differences among cultivars in terms of their reaction to WB on all leaves and spikes (Table 2). In location 2, there were differences in WB severity over time in all plant organs, except for leaves F-6 and F-5 (Table 3). Differences in WB severity were found between experiments in all plant organs except for leaf F-6 (Table 3). There were cultivar differences in terms of their reaction to $\mathrm{W}_{\mathrm{L}} \mathrm{B}$ (all leaves) and $\mathrm{W}_{\mathrm{S}} \mathrm{B}$ (Table 3 ). For both locations, there were substantial interactions among cultivar, DAE, and experiments on plant organ severity (Tables 2 and 3 ).

Temporal modeling. Ordinary least square regression performed on disease progress data for all 10 cultivars by location and experiment showed that overall the logistic model provided an adequate description of WB DPCs. In location 1, experiment 1, the logistic model best described the curves of all 10 cultivars $\left(R^{2}=0.75-0.96\right.$; $R M S E=$ 0.67-2.63). On experiment 2, the logistic model described seven curves $\left(\mathrm{R}^{2}=0.88-0.93 ; \mathrm{RMSE}=1.06-2.44\right)$, and the Gompertz model described three curves $\left(\mathrm{R}^{2}=0.91-0.94\right.$; $\mathrm{RMSE}=$ 0.13-0.23) (Table 5). In location 2, experiment 1, the logistic model best described the curves for all 10 cultivars $\left(\mathrm{R}^{2}=0.63-0.92\right.$; RMSE

Table 6. Summary of statistical parameters and Akaike's information criterium (AIC) and $\Delta$ AIC of linearized logistic and Gompertz models for 10 cultivars at two locations in Bolivia in 2018-2019

\begin{tabular}{|c|c|c|c|c|c|c|c|c|c|c|c|c|c|}
\hline \multirow[b]{3}{*}{ Cultivar } & \multirow[b]{3}{*}{ Model } & \multicolumn{12}{|c|}{ Location 1 (Bermejo) } \\
\hline & & \multicolumn{6}{|c|}{ Experiment 1} & \multicolumn{6}{|c|}{ Experiment 2} \\
\hline & & SSE $^{w}$ & Adj. $R^{2 \times}$ & $\mathrm{N}^{\mathbf{y}}$ & $\mathbf{K}^{\mathbf{z}}$ & AIC & $\Delta \mathrm{AIC}$ & SSE $^{w}$ & Adj. $R^{2 x}$ & $\mathrm{~N}^{\mathbf{y}}$ & $\mathbf{K}^{\mathbf{z}}$ & AIC & $\Delta \mathrm{AIC}$ \\
\hline \multirow[t]{2}{*}{ Atlax } & Logistic & 60.56 & 0.87 & 12 & 2 & 23.43 & 64.66 & 27.75 & 0.92 & 12 & 2 & 14.06 & 61.93 \\
\hline & Gompertz & 75.91 & 0.58 & 12 & 2 & 26.14 & 67.37 & 27.81 & 0.72 & 12 & 2 & 14.09 & 61.95 \\
\hline \multirow[t]{2}{*}{ Urubó } & Logistic & 5.80 & 0.94 & 12 & 2 & -4.72 & 36.52 & 5.42 & 0.93 & 12 & 2 & -5.54 & 42.33 \\
\hline & Gompertz & 0.28 & 0.92 & 12 & 2 & -41.24 & 0.00 & 0.16 & 0.94 & 12 & 2 & -47.87 & 0.00 \\
\hline \multirow[t]{2}{*}{ TBIO-Sossego } & Logistic & 8.53 & 0.94 & 12 & 2 & -0.10 & 41.14 & 17.40 & 0.88 & 12 & 2 & 8.46 & 56.32 \\
\hline & Gompertz & 2.00 & 0.85 & 12 & 2 & -17.47 & 23.77 & 0.53 & 0.94 & 12 & 2 & -33.44 & 14.43 \\
\hline \multirow[t]{2}{*}{ San Pablo } & Logistic & 8.52 & 0.92 & 12 & 2 & -0.10 & 41.13 & 11.18 & 0.88 & 12 & 2 & 3.15 & 51.01 \\
\hline & Gompertz & 0.45 & 0.90 & 12 & 2 & -35.40 & 5.84 & 0.40 & 0.89 & 12 & 2 & -36.94 & 10.92 \\
\hline \multirow[t]{2}{*}{ AN-120 } & Logistic & 4.51 & 0.95 & 12 & 2 & -7.74 & 33.50 & 9.74 & 0.89 & 12 & 2 & 1.49 & 49.36 \\
\hline & Gompertz & 0.31 & 0.91 & 12 & 2 & -39.94 & 1.29 & 0.33 & 0.91 & 12 & 2 & -39.16 & 8.71 \\
\hline \multirow[t]{2}{*}{ Motacú } & Logistic & 51.57 & 0.72 & 12 & 2 & 21.50 & 62.73 & 23.67 & 0.85 & 12 & 2 & 12.15 & 60.02 \\
\hline & Gompertz & 14.59 & 0.45 & 12 & 2 & 6.35 & 47.58 & 11.12 & 0.52 & 12 & 2 & 3.09 & 50.95 \\
\hline \multirow[t]{2}{*}{ BR-18 } & Logistic & 66.41 & 0.85 & 12 & 2 & 24.53 & 65.77 & 59.60 & 0.87 & 12 & 2 & 23.23 & 71.10 \\
\hline & Gompertz & 79.65 & 0.55 & 12 & 2 & 26.71 & 67.95 & 71.84 & 0.59 & 12 & 2 & 25.47 & 73.34 \\
\hline \multirow[t]{2}{*}{ TBIO-Mestre } & Logistic & 6.71 & 0.96 & 12 & 2 & -2.97 & 38.27 & 20.34 & 0.88 & 12 & 2 & 10.33 & 58.20 \\
\hline & Gompertz & 1.97 & 0.87 & 12 & 2 & -17.70 & 23.53 & 2.37 & 0.85 & 12 & 2 & -15.45 & 32.41 \\
\hline \multirow[t]{2}{*}{ TBIO-Mirante } & Logistic & 47.78 & 0.89 & 12 & 2 & 20.58 & 61.82 & 19.27 & 0.93 & 12 & 2 & 9.68 & 57.55 \\
\hline & Gompertz & 65.78 & 0.63 & 12 & 2 & 24.42 & 65.65 & 9.80 & 0.80 & 12 & 2 & 1.57 & 49.43 \\
\hline \multirow[t]{4}{*}{ TBIO-Alvorada } & Logistic & 69.01 & 0.85 & 12 & 2 & 24.99 & 66.23 & 19.77 & 0.92 & 12 & 2 & 9.99 & 57.86 \\
\hline & Gompertz & 77.57 & 0.59 & 12 & 2 & 26.39 & 67.63 & 7.02 & 0.81 & 12 & 2 & -2.43 & 45.44 \\
\hline & & \multicolumn{12}{|c|}{ Location 2 (Quirusillas) } \\
\hline & & \multicolumn{6}{|c|}{ Experiment 1} & \multicolumn{6}{|c|}{ Experiment 2} \\
\hline \multirow[t]{2}{*}{ Atlax } & Logistic & 8.64 & 0.92 & 9 & 2 & 3.64 & 32.03 & 35.20 & 0.82 & 9 & 2 & 16.27 & 34.52 \\
\hline & Gompertz & 3.50 & 0.78 & 9 & 2 & -4.50 & 23.90 & 34.87 & 0.65 & 9 & 2 & 16.19 & 34.44 \\
\hline \multirow[t]{2}{*}{ Urubó } & Logistic & 15.04 & 0.58 & 9 & 2 & 8.62 & 37.02 & 29.82 & 0.65 & 9 & 2 & 14.78 & 33.03 \\
\hline & Gompertz & 0.49 & 0.50 & 9 & 2 & -22.21 & 6.18 & 0.84 & 0.75 & 9 & 2 & -17.35 & 0.90 \\
\hline TBIO-Sossego & Logistic & 10.95 & 0.86 & 9 & 2 & 5.77 & 34.16 & 27.57 & 0.59 & 9 & 2 & 14.07 & 32.32 \\
\hline & Gompertz & 0.83 & 0.77 & 9 & 2 & -17.42 & 10.98 & 1.84 & 0.66 & 9 & 2 & -10.31 & 7.94 \\
\hline San Pablo & Logistic & 3.44 & 0.91 & 9 & 2 & -4.66 & 23.73 & 14.80 & 0.85 & 9 & 2 & 8.48 & 26.73 \\
\hline & Gompertz & 0.25 & 0.80 & 9 & 2 & -28.39 & 0.00 & 1.26 & 0.76 & 9 & 2 & -13.72 & 4.53 \\
\hline AN-120 & Logistic & 28.89 & 0.65 & 9 & 2 & 14.50 & 42.89 & 36.66 & 0.58 & 9 & 2 & 16.64 & 34.89 \\
\hline & Gompertz & 1.12 & 0.65 & 9 & 2 & -14.73 & 13.67 & 1.71 & 0.60 & 9 & 2 & -10.95 & 7.30 \\
\hline Motacú & Logistic & 33.08 & 0.70 & 9 & 2 & 15.72 & 44.11 & 30.49 & 0.76 & 9 & 2 & 14.98 & 33.23 \\
\hline & Gompertz & 2.04 & 0.68 & 9 & 2 & -9.36 & 19.03 & 1.83 & 0.75 & 9 & 2 & -10.35 & 7.90 \\
\hline BR-18 & Logistic & 37.34 & 0.74 & 9 & 2 & 16.81 & 45.20 & 5.54 & 0.93 & 9 & 2 & -0.36 & 17.88 \\
\hline & Gompertz & 3.02 & 0.72 & 9 & 2 & -5.82 & 22.58 & 1.32 & 0.86 & 9 & 2 & -13.28 & 4.97 \\
\hline TBIO-Mestre & Logistic & 43.79 & 0.67 & 9 & 2 & 18.24 & 46.63 & 3.08 & 0.96 & 9 & 2 & -5.64 & 12.61 \\
\hline & Gompertz & 2.40 & 0.69 & 9 & 2 & -7.91 & 20.48 & 0.76 & 0.87 & 9 & 2 & -18.25 & 0.00 \\
\hline TBIO-Mirante & Logistic & 35.04 & 0.91 & 9 & 2 & 16.23 & 44.63 & 55.11 & 0.79 & 9 & 2 & 20.31 & 38.56 \\
\hline & Gompertz & 53.25 & 0.74 & 9 & 2 & 20.00 & 48.40 & 52.74 & 0.71 & 9 & 2 & 19.91 & 38.16 \\
\hline TBIO-Alvorada & Logistic & 51.20 & 0.79 & 9 & 2 & 19.65 & 48.04 & 66.39 & 0.73 & 9 & 2 & 21.98 & 40.23 \\
\hline & Gompertz & 51.88 & 0.45 & 9 & 2 & 19.77 & 48.16 & 55.56 & 0.63 & 9 & 2 & 20.38 & 38.63 \\
\hline
\end{tabular}

w SSE: sum of squares of the error.

x Adj. $\mathrm{R}^{2}$ : adjusted $\mathrm{R}^{2}$.

y $\mathrm{N}$ : number of observations used in the model.

${ }^{\mathrm{z}} \mathrm{K}$ : number of parameters. 
$=0.70-2.70)$. In experiment 2 , the logistic model described seven curves $\left(\mathrm{R}^{2}=0.7-0.96 ; \mathrm{RMSE}=0.89-2.80\right)$, and Gompertz described three curves $\left(\mathrm{R}^{2}=0.64-0.77\right.$; $\left.\mathrm{RMSE}=0.34-0.51\right)$ (Table 5). The exponential and monomolecular models were deemed inadequate to describe any WB progress curve based on the statistical model selection criteria.

Delta AIC ( $\triangle \mathrm{AIC})$ for model selection between logistic and Gompertz in location 1 showed that, by experiment, Gompertz had lower average values (37.06 and 34.76) than logistic (51.18 and 56.57) for experiments 1 and 2, respectively. In location 2, Gompertz had lower values (21.34 and 14.48) than logistic (39.84 and 30.40) for experiments 1 and 2, respectively. However, the adjusted $\mathrm{R}^{2}$ $\left(\operatorname{Adj} R^{2}\right)$ was higher for logistic than Gompertz. At location 1, the logistic $\operatorname{Adj}^{2}$ values were $0.72-0.96$ in experiment 1 , and $0.85-0.92$ in experiment 2 , while the Gompertz $\mathrm{AdjR}^{2}$ values were $0.45-0.92$ in experiment 1 , and $0.52-0.94$ in experiment 2 . At location 2, the $\operatorname{logistic} \mathrm{Adj}^{2}$ values were $0.58-0.92$ experiment 1 , and $0.58-0.96$ in experiment 2, while the Gompertz $\operatorname{Adj}^{2}$ were $0.45-0.80$ in experiment 1 , and $0.60-0.87$ in experiment 2 (Table 6). Due to the above reasons for model selection criteria, logistic curves were selected to describe the temporal progress of WB.

Effect of cultivar resistance on disease and grain weight. According to the analysis of variance, in location 1, experiments 1 and 2 showed differences in tAUDPC among cultivars (Table 7). The lowest tAUDPCs were for cultivars Urubó and Motacú (tAUDPC $=107.50-468.90)$, and the highest values were for Atlax and TBIO-Mirante (AUDPC $=1,687.23-1,873.53$ ). The logistic apparent infection rates $\left(r_{L^{*}}\right)$ were different among cultivars in the two experiments, and the lowest rates were for cultivars Urubó and AN-120 (0.14-0.15 unit day $\left.{ }^{-1}\right)$, and the highest rates were for cultivars for Atlax, TBIO-Mirante, and TBIO-Alvorada $\left(0.29-0.34\right.$ unit day $\left.^{-1}\right)$. For final disease severity $\left(Y_{\max }\right)$, in both experiments, the lowest value was for cultivar Urubó $\left(Y_{\max }=\right.$ 7.82-12.49\%), while cultivars Atlax and BR-18 had the highest values $\left(Y_{\max }=99.83-100 \%\right)$. Grain weight loss values were different among cultivars, in both experiments, and the lowest grain weights were for Atlax, TBIO-Mirante, and TBIO-Alvorada $\left(0.00 \mathrm{~g} 100\right.$-seeds $\left.^{-1}\right)$, while the highest grain weight was obtained with cultivar Urubó (16.52-17.49 g 100-seeds $^{-1}$ ). In location 2, experiments 1 and 2 showed differences in tAUDPC among cultivars (Table 7). In experiment 1, the lowest tAUDPC was for cultivars Urubó (tAUDPC $=6.95-126.63$ ), and the highest values for Atlax, TBIO-Mirante, and TBIO-Alvorada (tAUDPC $=763.63-1,416.59$ ). The logistic apparent infection rates $\left(r_{L^{*}}\right)$ were different among cultivars in the two experiments; the lowest rate was for cultivar Urubó, $\left(0.10-0.18\right.$ per unit day $\left.{ }^{-1}\right)$ and the highest rates were for cultivars for Atlax, TBIO-Mirante, and TBIO-Alvorada $\left(0.35-0.48\right.$ unit day $\left.{ }^{-1}\right)$. For final disease severity percentage $\left(Y_{\max }\right)$, in both experiments, the lowest value was for cultivar Urubó $\left(Y_{\max }=4.90-16.47 \%\right)$, while cultivars Atlax, TBIOMirante, and TBIO-Alvorada had the highest values $\left(Y_{\max }=\right.$ 92.70-100\%). Grain weight loss were different among cultivars in both experiments, and the lowest grain weights were for Atlax, TBIO-Mirante, and TBIO-Alvorada (0.00-2.90 g 100-seeds $^{-1}$ ), while the highest grain weight was for cultivars Urubó and AN120 (27.63-29.02 g 100-seeds $^{-1}$ ) (Table 7).

The tAUDPC differed by locations. Location 1 (Bermejo) had higher values than location 2 (Quirusillas). However, within each location, cultivars in each of the two established experiments followed similar trends regarding levels of wheat blast resistance (Fig. 2). In location 1, all 10 cultivars in both experiments showed different levels of 1AUDPC and sAUDPC $(P=$ $0.0063)$; leaf values were 15.31-1,443.77, and spike values were 97.28-1,553.80. In location 2, 1AUDPC values ranged from 0.00 to 669.29 and sAUDPC ranged from 12.05 to $1,521.46(P=$ 0.0003) (Fig. 3).

Correlation among disease parameters. Significant correlations existed among disease parameters in both experiments at each of the two locations. For location 1, tAUDPC, the infection rate $r_{L^{*}}$, and $Y_{\max }$ were negatively correlated with grain weight, $(\mathrm{r}=-0.79$, $-0.81, P<0.01),(\mathrm{r}=-0.81,-0.82, P<0.01)$, and $(\mathrm{r}=-0.90$,

Table 7. Main effect of resistance from 10 cultivars on wheat blast severity at two locations in Bolivia in 2018-2019w

\begin{tabular}{|c|c|c|c|c|c|c|c|c|}
\hline \multirow[b]{3}{*}{ Cultivar } & \multicolumn{8}{|c|}{ Location 1 (Bermejo) } \\
\hline & \multicolumn{2}{|c|}{ tAUDPC ${ }^{x}$} & \multicolumn{2}{|c|}{$r_{L^{*}}\left(\text { unit day }^{-1}\right)^{\mathrm{y}}$} & \multicolumn{2}{|c|}{$Y_{\max }(\%)^{\mathrm{z}}$} & \multicolumn{2}{|c|}{$\begin{array}{c}\text { Grain weight } \\
\left.\text { (g 100-seeds }^{-1}\right)\end{array}$} \\
\hline & $\operatorname{Exp} 1$ & $\operatorname{Exp} 2$ & $\operatorname{Exp} 1$ & $\operatorname{Exp} 2$ & $\operatorname{Exp} 1$ & $\operatorname{Exp} 2$ & $\operatorname{Exp} 1$ & $\operatorname{Exp} 2$ \\
\hline Atlax & $1,472.82 \mathrm{AB}$ & $1,873.53 \mathrm{~A}$ & $0.34 \mathrm{~A}$ & $0.31 \mathrm{~A}$ & $100.00 \mathrm{~A}$ & $99.83 \mathrm{~A}$ & $0.00 \mathrm{D}$ & $0.00 \mathrm{D}$ \\
\hline Urubó & $156.91 \mathrm{E}$ & $107.50 \mathrm{E}$ & $0.15 \mathrm{C}$ & $0.14 \mathrm{C}$ & $12.49 \mathrm{C}$ & $7.82 \mathrm{D}$ & $17.49 \mathrm{~A}$ & $16.52 \mathrm{~A}$ \\
\hline TBIO-Sossego & $779.10 \mathrm{C}$ & $672.97 \mathrm{CB}$ & $0.20 \mathrm{~B}$ & $0.18 \mathrm{~B}$ & $70.22 \mathrm{~B}$ & $44.74 \mathrm{C}$ & $13.13 \mathrm{~B}$ & $8.87 \mathrm{~B}$ \\
\hline San Pablo & $203.86 \mathrm{E}$ & 186.04 DE & $0.16 \mathrm{C}$ & $0.14 \mathrm{C}$ & $17.01 \mathrm{C}$ & $14.98 \mathrm{D}$ & $15.46 \mathrm{AB}$ & $14.76 \mathrm{~A}$ \\
\hline AN-120 & $157.39 \mathrm{E}$ & $184.73 \mathrm{DE}$ & $0.14 \mathrm{C}$ & $0.14 \mathrm{C}$ & $14.24 \mathrm{C}$ & $15.41 \mathrm{D}$ & $17.08 \mathrm{~A}$ & $16.19 \mathrm{~A}$ \\
\hline Motacú & $468.90 \mathrm{D}$ & $546.42 \mathrm{CD}$ & $0.20 \mathrm{~B}$ & $0.20 \mathrm{~B}$ & $96.75 \mathrm{~A}$ & $95.58 \mathrm{~A}$ & $0.00 \mathrm{D}$ & $0.00 \mathrm{D}$ \\
\hline BR-18 & $1,390.00 \mathrm{~B}$ & $1,604.28 \mathrm{~A}$ & $0.32 \mathrm{~A}$ & $0.33 \mathrm{~A}$ & $100.00 \mathrm{~A}$ & $100.00 \mathrm{~A}$ & $0.00 \mathrm{D}$ & $0.00 \mathrm{D}$ \\
\hline TBIO-Mestre & $900.44 \mathrm{C}$ & $1,027.39 \mathrm{~B}$ & $0.23 \mathrm{~B}$ & $0.22 \mathrm{~B}$ & $72.62 \mathrm{~B}$ & $75.51 \mathrm{~B}$ & $0.00 \mathrm{C}$ & $5.30 \mathrm{C}$ \\
\hline TBIO-Mirante & $1,687.23 \mathrm{~A}$ & $1,720.79 \mathrm{~A}$ & $0.34 \mathrm{~A}$ & $0.30 \mathrm{~A}$ & $100.00 \mathrm{~A}$ & $97.58 \mathrm{~A}$ & $0.00 \mathrm{D}$ & $0.00 \mathrm{D}$ \\
\hline \multirow[t]{2}{*}{ TBIO-Alvorada } & $1,460.32 \mathrm{AB}$ & $1,626.55 \mathrm{~A}$ & $0.34 \mathrm{~A}$ & $0.29 \mathrm{~A}$ & $100.00 \mathrm{~A}$ & $94.75 \mathrm{AB}$ & $0.00 \mathrm{D}$ & $0.00 \mathrm{D}$ \\
\hline & \multicolumn{8}{|c|}{ Location 2 (Quirusillas) } \\
\hline Atlax & $763.63 \mathrm{~B}$ & $1,366.35 \mathrm{~A}$ & $0.35 \mathrm{~B}$ & $0.35 \mathrm{~A}$ & $92.70 \mathrm{~A}$ & $100.00 \mathrm{~A}$ & $2.90 \mathrm{D}$ & $0.00 \mathrm{~F}$ \\
\hline Urubó & $6.95 \mathrm{E}$ & $126.63 \mathrm{E}$ & $0.10 \mathrm{E}$ & $0.18 \mathrm{DE}$ & $4.90 \mathrm{E}$ & $16.47 \mathrm{D}$ & $29.02 \mathrm{~A}$ & $27.63 \mathrm{AB}$ \\
\hline TBIO-Sossego & $97.46 \mathrm{D}$ & $305.96 \mathrm{DE}$ & $0.20 \mathrm{C}$ & $0.18 \mathrm{E}$ & $27.50 \mathrm{D}$ & $47.91 \mathrm{CB}$ & $25.20 \mathrm{AB}$ & $23.97 \mathrm{BC}$ \\
\hline San Pablo & $20.41 \mathrm{E}$ & $283.49 \mathrm{DE}$ & $0.11 \mathrm{E}$ & $0.21 \mathrm{CDE}$ & $6.37 \mathrm{E}$ & $33.07 \mathrm{C}$ & $26.65 \mathrm{AB}$ & $25.62 \mathrm{ABC}$ \\
\hline $\mathrm{AN}-120$ & $72.26 \mathrm{D}$ & $131.82 \mathrm{E}$ & $0.18 \mathrm{CD}$ & $0.19 \mathrm{DE}$ & $13.49 \mathrm{E}$ & $32.70 \mathrm{CD}$ & $28.33 \mathrm{~A}$ & $29.00 \mathrm{~A}$ \\
\hline Motacú & $221.81 \mathrm{CDE}$ & $328.68 \mathrm{D}$ & $0.22 \mathrm{C}$ & $0.24 \mathrm{~B}$ & $37.71 \mathrm{D}$ & $38.47 \mathrm{C}$ & $23.75 \mathrm{~B}$ & $19.68 \mathrm{D}$ \\
\hline BR-18 & $451.15 \mathrm{C}$ & $626.02 \mathrm{C}$ & $0.25 \mathrm{C}$ & $0.22 \mathrm{BC}$ & $55.63 \mathrm{~B}$ & $62.32 \mathrm{~B}$ & $17.35 \mathrm{C}$ & $14.69 \mathrm{E}$ \\
\hline TBIO-Mestre & $306.20 \mathrm{CD}$ & $330.83 \mathrm{D}$ & $0.23 \mathrm{C}$ & $0.22 \mathrm{BCD}$ & $50.44 \mathrm{CB}$ & $45.31 \mathrm{CB}$ & $25.21 \mathrm{AB}$ & $22.74 \mathrm{DC}$ \\
\hline TBIO-Mirante & $1,167.57 \mathrm{~A}$ & $1,416.59 \mathrm{~A}$ & $0.48 \mathrm{~A}$ & $0.36 \mathrm{~A}$ & $100.00 \mathrm{~A}$ & $100.00 \mathrm{~A}$ & $0.00 \mathrm{D}$ & $0.00 \mathrm{~F}$ \\
\hline TBIO-Alvorada & 839.91 B & $1,191.74 \mathrm{~B}$ & $0.41 \mathrm{~B}$ & $0.37 \mathrm{~A}$ & $100.00 \mathrm{~A}$ & $100.00 \mathrm{~A}$ & $0.00 \mathrm{D}$ & $0.00 \mathrm{~F}$ \\
\hline
\end{tabular}

\footnotetext{
${ }^{w}$ Values with the same uppercase letter in the same column are means with no significant difference $(P>0.05)$.

$x$ tAUDPC: total AUDPC.

y $r_{L^{*}}$ : logistic rate.

${ }^{\mathrm{z}} Y_{\max }$ : final disease severity.
} 
$-0.96, P<0.01)$, respectively. For location 2, tAUDPC, infection rate $r_{L^{*}}$, and $Y_{\max }$ were also negatively correlated with grain weight, $(\mathrm{r}=$ $-0.88,-0.95, P<0.01),(\mathrm{r}=-0.87,-0.90, P<0.01)$, and $(\mathrm{r}=-0.89$, $-0.92, P<0.01)$. In location 1 , there were negative correlations between disease parameters, tAUDPC, $r_{L^{*}}$, and $Y_{\max }(P<0.05)$ (Table 8).

Regression between grain weight and tAUDPC to predict grain weight loss showed that slope coefficient as estimator of grain weight loss was higher in location 2 (Quirusillas) $\left(-0.021,-0.025 \mathrm{~g}^{-1}\right)$ than in location 1 (Bermejo) $\left(-0.008,-0.010 \mathrm{~g}^{-1}\right)$ for each unit of tAUDPC (Fig. 4).

\section{Discussion}

This study provides clear evidence of the relevance of plant disease epidemiological criteria to support breeding tactics against WB. As a starting point, cultivar resistance levels influenced the dynamics of $\mathrm{W}_{\mathrm{L}} \mathrm{B}$ and $\mathrm{W}_{\mathrm{S}} \mathrm{B}$ development in various environments. In addition, epidemiological parameters associated with WB resistance were critical for selection and deployment of germplasm. In general, WB symptoms developed over time on all plant organs, starting from the lower canopy in all cultivars. At both locations, there were differences in disease severity between experiments. These differences were likely due to the distance between the MoT inoculum source and individual plots. According to Salgado et al. (unpublished), MoT infections initially developed at the lower canopy, where leaf blast severity reached higher values compared with upper canopy leaves. This might be due to senescence of lower leaves (Góngora-Canul et al.

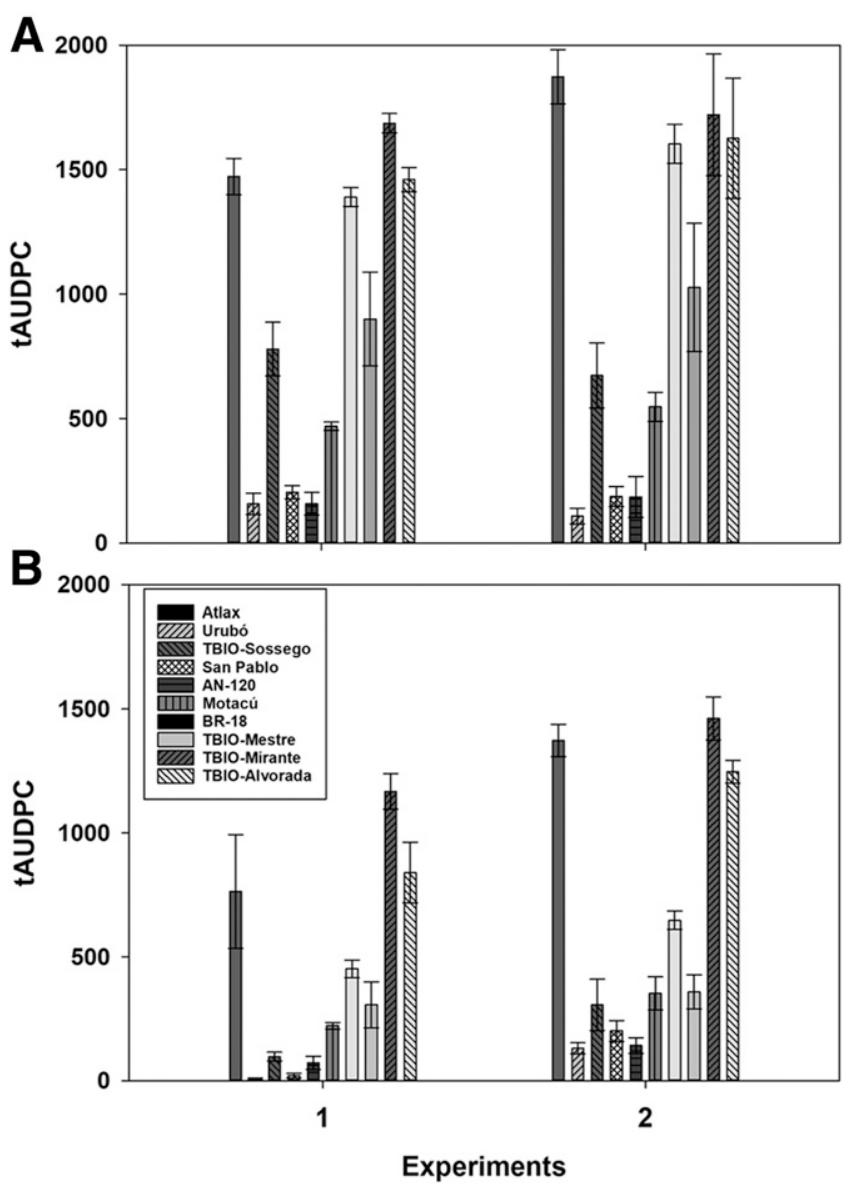

Fig. 2. Total area under disease progress curve (tAUDPC) of 10 wheat cultivars with different levels of wheat blast resistance considering leaves and spikes. Error bars indicate standard error of the mean. Two experiments were established at two locations in Bolivia, Bermejo (A) and Quirusillas (B), during the 2018-2019 wheat growing season.
2020). In addition, a massive influx of MoT conidia from the lower canopy and subsequent compounding secondary infection cycles in the upper canopy may have occurred and caused high WB severity (Góngora-Canul et al. 2020; Salgado et al., unpublished).

Regardless of environment, the logistic model best described WB progress. In both locations, the logistic model described 17 out of 20 DPCs $(85 \%)$ and the Gompertz model described three out of $20(15 \%)$. Similar results were found by Góngora-Canul et al. (2020) and Mohapatra et al. (2008). $\Delta$ AIC for model selection showed that Gompertz had lower values than the logistic model in all experiments, but the logistic model had higher $\operatorname{Adj}^{2}$ values. The latter indicated that the logistic model better fit the data. The $\mathrm{AdjR}^{2}$ values alone can consistently choose complex models compared with selection based on AIC values, and those models can reproduce better empirical datasets than those chosen by AIC (Gayawan and Ipinyomi 2009). For those reasons, the logistic model was deemed more adequate and selected to describe the temporal progress of WB.

In both locations, there were significant differences in tAUDPC of blast severity among the 10 spring wheat cultivars of interest. Cultivars Urubó, San Pablo, and TBIO-Sossego had the lowest, and cultivars Atlax, TBIO-Mirante, and TBIO-Alvorada had the highest values of tAUDPC, respectively. The apparent infection rate $\left(r_{L^{*}}\right)$ (units day $^{-1}$ ) of linearized logistic model was low for cultivars Urubó, San Pablo, and TBIO-Sossego and the higher $r_{L^{*}}$ values were for Atlax, TBIO-Mirante, and TBIO-Alvorada. In general, more tAUDPC led to higher apparent infection rates; however, cultivar Motacú was the exception. This cultivar had low values of tAUDPC, but high $r_{L^{*}}$, because it provided sufficient resistance to $\mathrm{W}_{\mathrm{L}} \mathrm{B}$ but not enough for $\mathrm{W}_{\mathrm{S}} \mathrm{B}$ (Fig. 3). This early maturity cultivar, which became popular in Bolivia over the last few years (Vales et al. 2018), is known for its moderate to insufficient $\mathrm{W}_{\mathrm{S}} \mathrm{B}$ resistance reaction, which can vary by location and season (Cruz et al. 2016a; Vales et al. 2018). This was the only case in which low tAUDPC did not necessarily imply a lower $r_{L^{*}}$.

Pratt et al. (1993) reported that AUDPC was more important for cultivar selection than rate, since AUDPC as a measure of quantitative disease resistance entails repeated disease assessment. However, Jeger and Viljanen-Rollinson (2001) mention that the effect of resistance should be expressed in terms of rate parameter ('rate-reducing' effect) and not based on the asymptotic level of disease (maximum amount of disease level without increase) in adult plants. Fry (1978) reported that AUDPC is more reliable than $r^{*}$ and final disease severity $\left(Y_{\max }\right)$ for describing cultivar resistance, arguing that $Y_{\max }$ was useful only if the epidemic did not progress to completion or near completion by the end of the season, and that $r^{*}$ is influenced by weather. Regarding $Y_{\max }$, cultivars Urubó, AN120 , and San Pablo had the lowest values, up to $4.9 \%$ severity. Atlax, TBIO-Mirante, and TBIO-Alvorada cultivars had values of up to $100 \%$ severity. The trend general is that the higher the $Y_{\max }$ value, the higher the AUDPC and $r_{L^{*}}$ values. Final disease severity evaluation is a practical and cheap estimator when many cultivars are being evaluated for resistance. However, using final disease severity alone could be risky if disease pressure is not high and temporal dynamics are not understood in terms of disease onset and temporal rates of progress, as it was the example of cultivar Motacú. There were significant differences in grain weight $\left(\mathrm{g} 100-\right.$ seeds $\left.^{-1}\right)$. Overall, cultivars in location 1 had less grain weight than in location 2. Cultivars that had low or zero grain weight were Atlax, TBIO-Mirante, and TBIO-Alvorada, and cultivars with higher grain weight were Urubó, AN-120, and San Pablo. The reduction of grain weight affected by disease severity (tAUDPC, $r_{L^{*}}$, and $Y_{\max }$ ) was due to reduction of leaf green area in early phases of the epidemic and by the infection of the spike resulting in no grain formation or shriveled grain.

Differences were observed among cultivars in their combined reaction to $\mathrm{W}_{\mathrm{L}} \mathrm{B}$ and $\mathrm{W}_{\mathrm{S}} \mathrm{B}$ in both locations. Cultivars that had lowest tAUDPC such as Urubó, San Pablo, and TBIO-Sossego had the 
lowest 1AUDPC and sAUDPC, respectively. Susceptible cultivars such Atlax, TBIO-Mirante, and TBIO-Alvorada had the highest tAUDPC as well as 1AUDPC and sAUDPC. In contrast, cultivar Motacú had the lowest 1AUDPC but not the lowest sAUDPC. Differences in reaction to WB according to plant age and organs have been previously reported (Cruppe et al. 2020; Cruz et al. 2012; Martínez et al. 2019). WB disease can be found in spike and leaf organs, and weak correlations have been reported between seedling and adult plant resistance across multiple cultivars (Cruppe et al 2020; Cruz et al. 2012; Maciel et al. 2014). Different mechanisms
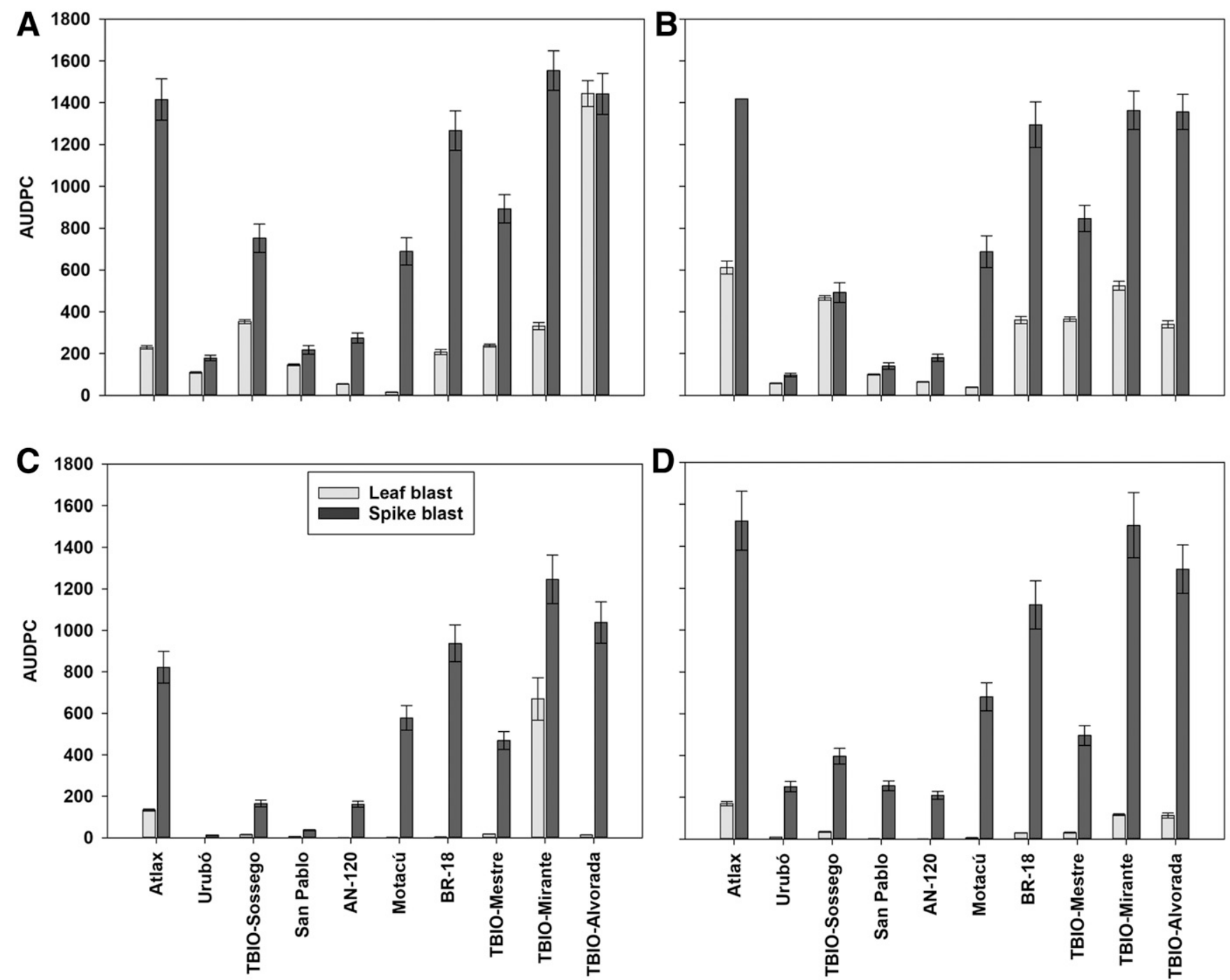

Fig. 3. Area under disease progress curve of wheat leaf (IAUDPC) and spike (sAUDPC) blast of 10 wheat cultivars in location 1 (Bermejo) according to experiments 1 (A) and 2 (B). Results for location 2 (Quirusillas) include experiment 1 (C) and experiment 2 (D). Error bars indicate standard error of the mean. Experiments were conducted in Bolivia during the 2018-2019 wheat growing season.

Table 8. Pearson correlation coefficients for total area under disease progress curve (tAUDPC), linearized logistic infection rate of disease progress $\left(r_{L^{*}}\right)$, final WB disease severity $\left(Y_{\max }\right)$, and grain weight in two locations in Bolivia, 2018-2019z

\begin{tabular}{|c|c|c|c|c|c|c|c|c|}
\hline & \multicolumn{8}{|c|}{ Location 1 (Bermejo) } \\
\hline & \multicolumn{4}{|c|}{ Experiment 1} & \multicolumn{4}{|c|}{ Experiment 2} \\
\hline & tAUDPC & $r_{L^{*}}\left(\right.$ day $\left.^{-1}\right)$ & $Y_{\max }(\%)$ & $\begin{array}{c}\text { Grain weight } \\
\text { (g 100-seeds }^{-1} \text { ) }\end{array}$ & tAUDPC & $r_{L^{*}}\left(\right.$ day $\left.^{-1}\right)$ & $Y_{\max }(\%)$ & $\begin{array}{c}\text { Grain weight } \\
\left.\text { (g 100-seeds }^{-1}\right)\end{array}$ \\
\hline tAUDPC & $\ldots$ & $0.972 * *$ & $0.861 * *$ & $-0.791 * *$ & $\ldots$ & $0.951 * *$ & $0.852 * *$ & $-0.818 * *$ \\
\hline$r_{L^{*}}\left(\right.$ per unit day $\left.{ }^{-1}\right)$ & & $\ldots$ & $0.851 * *$ & $-0.816^{* *}$ & & $\ldots$ & $0.864 * *$ & $-0.828 * *$ \\
\hline$Y_{\max }(\%)$ & & & $\ldots$ & $-0.906^{* *}$ & & & $\ldots$ & $-0.963 * *$ \\
\hline \multirow[t]{3}{*}{ Grain weight (g 100-seeds ${ }^{-1}$ ) } & & & & $\ldots$ & & & & $\ldots$ \\
\hline & \multicolumn{8}{|c|}{ Location 2 (Quirusillas) } \\
\hline & \multicolumn{4}{|c|}{ Experiment 1} & \multicolumn{4}{|c|}{ Experiment 2} \\
\hline tAUDPC & $\ldots$ & $0.959 * *$ & $0.912 * *$ & $-0.888 * *$ & $\ldots$ & $0.930 * *$ & $0.940 * *$ & $-0.951 * *$ \\
\hline$r_{L^{*}}\left(\right.$ per unit day $\left.{ }^{-1}\right)$ & & $\ldots$ & $0.926^{* *}$ & $-0.873^{* *}$ & & $\ldots$ & $0.880 * *$ & $-0.908 * *$ \\
\hline$Y_{\max }(\%)$ & & & $\ldots$ & $-0.898 * *$ & & & $\ldots$ & $-0.920 * *$ \\
\hline Grain weight (g 100-seeds $\left.{ }^{-1}\right)$ & & & & $\ldots$ & & & & $\ldots$ \\
\hline
\end{tabular}

$\mathrm{z} * *: P<0.01$, and $*: P<0.05$. 
for WB resistance (Cruz and Valent 2017) might exist in leaves and spikes of cultivar Motacú.

There were significant $(P<0.01)$ positive correlations among tAUDPC, apparent infection rates $\left(r^{*}\right)$, and final disease severity $\left(Y_{\text {max }}\right)$ in both locations, indicating differences in resistance among cultivars. However, the correlation was higher between tAUDPC and $r_{L^{*}}$ than tAUDPC and $Y_{\max }$. This indicated that tAUDPC or $r^{*}$ can be chosen for cultivar selection in a WB breeding program. However, $Y_{\max }$ could be a cheap and easy estimator, since tAUDPC and $r_{L^{*}}$ are tedious and labor intensive due to repeated measurements that are needed (Smith et al. 1988). tAUDPC, $r_{L^{*}}$, and $Y_{\max }$ had significant negative correlations with grain weight $\left(\mathrm{g} 100\right.$ seeds $\left.^{-1}\right)$ at both locations $(\mathrm{r}>0.79)(P<0.01)$. However, $Y_{\max }$ had the highest correlation with grain weight, indicating that it can be used as a predictor in a single-point grain-weight loss model (critical-point) for WB since it requires less data over time. tAUDPC and $r_{L^{*}}$ can also be used as predictors in a multiple-point grain-weight loss WB model (multiple-point); however, they require several disease assessments over time, and that may not be feasible in some cases. Multiple-point models are usually more accurate than critical-point models because of their more thorough description of the epidemic (Madden 1983).

The development and release of new cultivars occur in the face of multiple breeding objectives such as improved yield, quality, and disease and drought resistance. In certain countries, the selection of newly released cultivars is based less on resistance to blast than on early maturity (Vales et al. 2018). Motacú, an early maturity cultivar, was preferred in Bolivian breeding programs, although it had insufficient resistance levels to $\mathrm{W}_{\mathrm{S}} \mathrm{B}$. The decision to deploy Motacú was risky because the WB temporal dynamics were not understood at the time of selection. Today, we understand that this widely deployed cultivar in Bolivia is characterized by moderate to insufficient $W_{S} B$ resistance, which can vary depending on location and year. Wheat cultivars resistant to $\mathrm{W}_{\mathrm{L}} \mathrm{B}$ can help reduce the rate of WB epidemics. Cultivars with better performance for both $\mathrm{W}_{\mathrm{L}} \mathrm{B}$ and $\mathrm{W}_{\mathrm{S}} \mathrm{B}$ resistance should be preferred over those that only perform well for either leaf blast or spike blast. There is evidence that $2 \mathrm{NS}$-based cultivars are not immune to $\mathrm{W}_{\mathrm{S}} \mathrm{B}$ but this translocation provides cultivars some ability to deal with a given MoT load (Cruz et al. 2016b).

This is the first study that provides evidence that germplasm with different resistance background can influence the dynamics of $\mathrm{W}_{\mathrm{L}} \mathrm{B}$ and $\mathrm{W}_{\mathrm{S}} \mathrm{B}$ development under multiple environments. Moreover, this study provides enough evidence and strongly supports the use of epidemiological parameters such as AUDPC, $r_{L^{*}}$, and $Y_{\max }$, which could be used to help pathologists and breeders better select germplasm for WB resistance. Given the threat of the introduction of the disease to new areas of the world, a better epidemiological understanding of WB development on novel genetic resources is needed.

\section{Acknowledgments}

The authors wish to thank the Asociación de Productores de Oleaginosas y Trigo (ANAPO) for support with the experiments conducted in Bolivia and BiotrigoGenética for providing seeds. The authors wish to acknowledge Lidia Calderón, Jorge Cuellar, Darwin Coimbra, Franklin Cortez, and Diego F. Baldelomar for their help with field activities.
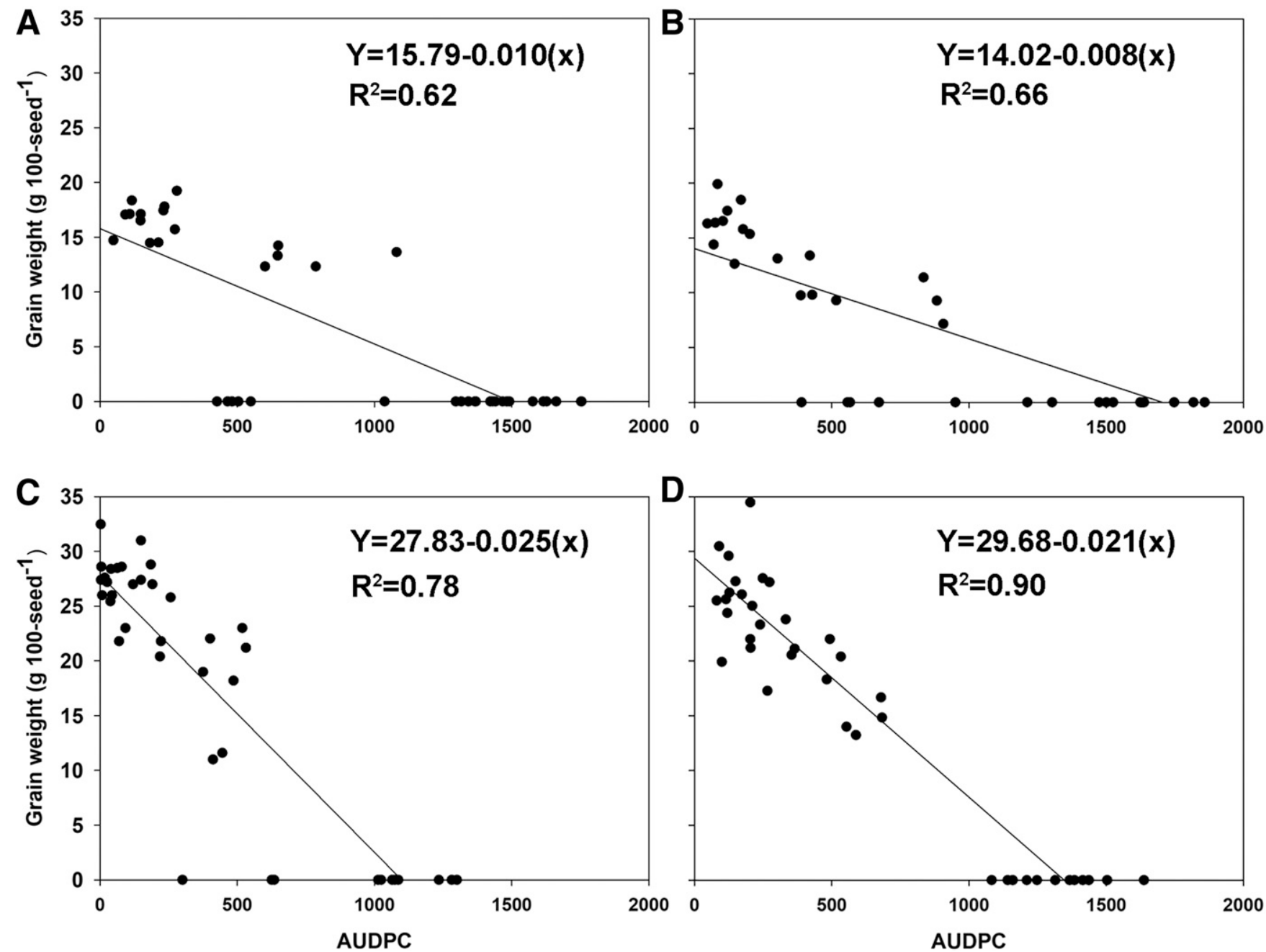

Fig. 4. Linear regression between grain weight and wheat blast severity of 10 cultivars. A and $\mathbf{B}$, experiments in location 1 (Bermejo). C and $\mathbf{D}$, experiments in location 2 (Quirusillas). Experiments were conducted in Bolivia during the 2018-2019 wheat growing season. 


\section{Literature Cited}

Arruda, M., Bueno, C., Zamprogno, K., Lavorenti, N., and Urashima, A. 2005. Reação do trigo à Magnaporthe grisea nos diferentes estádios de desenvolvimento. Fitopatol. Bras. 30:121-126.

Baldelomar, D., Cardenas, S., and Quispe, K. 2015. Caracterización de genotipos de trigo (Triticum aestivum) con resistencia a piricularia durante el verano 2014/2015 en Quirusillas, Santa Cruz, Bolivia. Info INIAF 1(6): 17-21.

Barea, G., and Toledo, J. 1996. Identificación y zonificación de piricularia o bruzone (Pyricularia oryzae) en el cultivo del trigo en el dpto. de Santa Cruz. CIAT. Pages 76-86 in: Informe Técnico. Proyecto de Investigación Trigo, Santa Cruz, Bolivia.

Cabrera, M. G., and Gutiérrez, S. 2007. Primer registro de Pyricularia grisea en cultivos de trigo del NE de Argentina. Depto. Protección Vegetal, Facultad de Ciencias Agrarias, Universidad Nacional del Nordeste. http:// www.agr.unne.edu.ar/images/documentos/extension/Res2007/SanVegetal/ SanVegetal_06.pdf

Campbell, C. L., and Madden, L. V. 1990. Introduction to plant disease epidemiology. John Wiley \& Sons, New York.

Cruppe, G., Cruz, C. D., Peterson, G., Pedley, K., Asif, M., Fritz, A., Calderon, L., Lemes da Silva, C., Todd, T., Kuhnem, P., Singh, P. K., Singh, R. P., Braun, H., Barma, N. C. D., and Valent, B. 2020. Novel sources of wheat head blast resistance in modern breeding lines and wheat wild relatives. Plant Dis. 104: 35-43.

Cruz, C. D., Bockus, W. W., Stack, J. P., Tang, X., Valent, B., Pedley, K. F., and Peterson, G. L. 2012. Preliminary assessment of resistance among U.S. wheat cultivars to the Triticum pathotype of Magnaporthe oryzae. Plant Dis. 96: 1501-1505.

Cruz, C. D., Kiyuna, J., Bockus, W. W., Todd, T. C., Stack, J. P., and Valent, B. 2015. Magnaporthe oryzae conidia on basal wheat leaves as a potential source of wheat blast inoculum. Plant Pathol. 64:1491-1498.

Cruz, C. D., Magarey, R. D., Christie, D. N., Fowler, G. A., Fernandes, J. M., Bockus, W. W., Valent, B., and Stack, J. P. 2016a. Climate suitability for Magnaporthe oryzae Triticum pathotype in the United States. Plant Dis. 100: 1979-1987.

Cruz, C. D., Peterson, G. L., Bockus, W. W., Kankanala, P., Dubcovsky, J., Jordan, K. W., Akhunov, E., Chumley, F., Baldelomar, F. D., and Valent, B. 2016b. The 2NS translocation from Aegilops ventricosa confers resistance to the Triticum pathotype of Magnaporthe oryzae. Crop Sci. 56:990-1000.

Cruz, C. D., Santana, F. M., Todd, T. C., Maciel, J. L. N., Kiyuna, J., Baldelomar, D. F., Cruz, A. P., Lau, D., Seixas, C. S., Goulart, A. C. P., Sussel, A. A., Schipanski, C. A., Chagas, D. F., Coelho, M., Montecelli, T. D. N., Utiamada, C., Custódio, A. P., Rivadeneira, M. G., Bockus, W. W., and Valent, B. 2019. Multi-environment assessment of fungicide performance for managing wheat head blast (WHB) in Brazil and Bolivia. Trop. Plant Pathol. 44:183-191.

Cruz, C. D., and Valent, B. 2017. Wheat blast disease: danger on the move. Trop. Plant Pathol. 42:210-222.

Fry, W. E. 1978. Quantification of general resistance of potato cultivars and fungicide effects for integrated control of potato late blight. Phytopathology 68:1650-1655

Gayawan, E., and Ipinyomi, R. A. 2009. A comparison of Akaike, Schwarz and R square criteria for model selection using some fertility models. Aust. J. Basic Appl. Sci. 3:3524-3530.

Gomes, D. P., Rocha, V. S., Rocha, J. R. A. S. C., Souza, M. A., and Pereira, O. L. 2019. Progresso temporal da brusone do trigo em função do inóculo primário, da aplicação defungicida e da resistência dos genótipos. Summa Phytopathol. 45:50-58.

Góngora-Canul, C., Salgado, J., Singh, D., Cruz, A., Cotrozzi, L., Couture, J. J., Rivadeneira, M. G., Cruppe, G., Valent, B., Todd, T., Poland, J., and Cruz, C. D. 2020. Temporal dynamics of wheat blast epidemics and agreement between remotely sensed data measurements and visual estimations of wheat spike blast (WSB) under field conditions. Phytopathology 110:393-405.

Goulart, A., and Paiva, F. 1992. Incidência da brusone (Pyricularia oryzae) em diferentes cultivares de trigo (Triticum aestivum) em condições de campo. Fitopatol. Bras. 17:321-325.
Igarashi, S. 1990. Update on wheat blast (Pyricularia oryzae) in Brazil. Pages 480-483 in: Proc. Int. Conf. Wheat for Nontraditional Warm Areas. D. Saunders, ed. CIMMYT, Mexico, D.F.

Igarashi, S., Utiamada, C. M., Igarashi, L. C., Kazuma, A. H., and Lopes, R. S. 1986. Pyricularia em trigo. 1. Ocorrência de Pyricularia sp. no estado do Paraná. Fitopatol. Bras. 11:351-352.

Jeger, M. J., and Viljanen-Rollinson, S. L. H. 2001. The use of the area under the disease-progress curve (AUDPC) to assess quantitative disease resistance in crop cultivars. Theor. Appl. Genet. 102:32-40.

Kaplan, S., and Gürcan, E. K. 2018. Comparison of growth curves using non-linear regression function in Japanese quail. J. Appl. Anim. Res. 46:112-117.

Maciel, J. L. N., Ceresini, P. C., Castroagudin, V. L., Zala, M., Kema, G. H. J., and McDonald, B. A. 2014. Population structure and pathotype diversity of the wheat blast pathogen Magnaporthe oryzae 25 years after its emergence in Brazil. Phytopathology 104:95-107.

Madden, L. V. 1983. Measuring and modeling crop losses at the field level. Phytopathology 73:1591-1596.

Madden, L. V., Hughes, G., and van den Bosch, F., eds. 2007. The Study of Plant Disease Epidemics. APS Press, St. Paul, MN.

Malaker, P. K., Barma, N. C. D., Tiwari, T. P., Collis, W. J., Duveiller, E., Singh, P. K., Joshi, A. K., Singh, R. P., Braun, H. J., Peterson, G. L., Pedley, K. F., Farman, M. L., and Valent, B. 2016. First report of wheat blast caused by Magnaporthe oryzae pathotype triticum in Bangladesh. Plant Dis. 100:2330.

Martínez, S. I., Sanabria, A., Fleitas, M. C., Consolo, V. F., and Perello, A. 2019. Wheat Blast: Aggressiveness of isolates of Pyricularia oryzae and effect on grain quality. J. King Saud Univ. 31:150-157.

Mazerolle, M. J. 2006. Improving data analysis in herpetology: using Akaike's Information Criterion (AIC) to assess the strength of biological hypotheses. Amphib.-Reptil. 27:169-180.

Mohapatra, N. K., Mukherjee, A. K., Suriya Rao, A. V., and Nayak, P. 2008. Disease progress curve in the rice pathosystem compared with the logistic and Gompertz model. ARPN J. Agric. Biol. Sci. 3:28-37.

Pratt, R. C., Adipala, E., and Lipps, P. E. 1993. Characterization of racenonspecific resistance to Exserohilum turcicum Races 0 and 1 in Maize $\mathrm{OhS}_{10} \mathrm{~S}_{1}$ Progenies. Plant Dis. 77:1227-1232.

Prestes, A., Arendt, P., Fernandes, M., and Scheeren, P. 2007. Resistance to Magnaporthe grisea among Brazilian wheat genotypes. Pages 119-133 in: Wheat Production in Stressed Environments. H. T. Buck, J. E. Nisi, and N. Salomón, eds. Springer, Mar del Plata, Argentina.

Priestley, R. H. 1978. Detection of increased virulence in populations of wheat yellow rust. Page 64 in: Plant Disease Epidemiology. P. R. Scott and A. Bainbridge, eds. Blackwell, Oxford.

Raji, C. A., Tarzwell, R., Pavel, D., Schneider, H., Uszler, M., Thornton, J., van Lierop, M., Cohen, P., Amen, D. G., and Henderson, T. 2014. Clinical utility of SPECT neuroimaging in the diagnosis and treatment of traumatic brain injury: a systematic review. PLoS One 9:e91088.

Renner-Martin, K., Kühleitner, M., Brunner, N., and Hagmüller, W. 2016. AICbased selection of growth models: The case of piglets from organic farming. Open J. Model. Simul. 4:17-23.

Smith, V. L., Campbell, C. L., Jenkins, S. F., and Benson, D. M. 1988. Effect of host density and number of disease foci on epidemics of southern blight of processing carrot. Phytopathology 78:595-600.

Urashima, A., Lavorenti, N. A., Goulart, A. C. P., and Mehta, Y. R. 2004. Resistance spectra of wheat cultivars and virulence diversity of Magnaporthe grisea isolates in Brazil. Fitopatol. Bras. 29:511-518.

Urashima, A. S., Hashimoto, Y., Don, L. D., Kusaba, M., Tosa, Y., Nakayashiki, H., and Mayama, S. 1999. Molecular analysis of the wheat blast population in Brazil with a homologue of retrotransposon MGR583. Ann. Phytopathol. Soc. Jpn. 65:429-436.

Vales, M., Anzoátegui, T., Huallpa, B., and Cazon, M. I. 2018. Review on resistance to wheat blast disease (Magnaporthe oryzae Triticum) from the breeder point-of-view: use of the experience on resistance to rice blast disease. Euphytica 214:1.

Viedma, L. Q. 2005. Wheat blast occurrence in Paraguay. Phytopathology 95: S152. 